\title{
Quality Prediction Modeling of a Preform Fastener Process using Fuzzy Logic and DEFORM Simulation
}

\author{
Suthep Butdee ${ }^{1 *}$, Uten Khanawapee ${ }^{2}$ \\ ${ }^{1}$ Laboratory Department of Production Engineering, Faculty of Engineering, King Mongkut's University of \\ Technology North Bangkok, Pracharaj 1, Bang Sue, Bangkok, 10800, Thailand \\ ${ }^{2}$ Department of Mechanical Engineering Technology, College of Industrial Technology, King Mongkut's \\ University of Technology North Bangkok, Pracharaj 1, Bang Sue, Bangkok, 10800, Thailand
}

\begin{abstract}
Quality is the most important aspect of fastener production for maintaining competitiveness and customer satisfaction. Nowadays, the quality control process is uncertain and complicated. Cold forging is used to produce preform fasteners via various processes. As a traditional measure of cold forging, quality prediction can be done using the normal probability method, but this approach is not effective or accurate enough. This paper proposes a novel quality prediction for preform fasteners using a fuzzy inference system and DEFORM simulation using an engineering software called DEFORM ${ }^{\circledR}$ system that can analyze metal forming. Multi-factors and criteria are considered, consisting of deformability, defects, stress, and time. The developed modeling can predict the cold forging quality at the stage of product design, which can associate decision making with production control. It can eliminate defects and reduce cost and time to rework. The case study is illustrated on the cold heading of stainless 341 using DEFORM, whereas the modeling is simulated by MATLAB.
\end{abstract}

Keywords: Cold forging stainless 341; DEFORM; Fuzzy logic; Preform fastener; Quality prediction

\section{Introduction}

Cold forging is used as a preform fastener production process; it involves several complex processes and parameters using punch and die sets that apply forces and heading speed. Several parameters and conditions are considered from design to production, including materials, machine capacity, punch and die design, and production processes. Fasteners are the most commonly used cold heading process in the automotive industry. Therefore, material flow is the main constraint under cold forging conditions; the difficulty of production when complex shapes are required is also an important factor. High-quality finished products are presently requested by customers. However, the quality of a new model cannot be ensured until the product is completely produced. As a result, it is necessary to develop quality prediction methodologies with multi-decision-making criteria using scientific methods, such as the analytic hierarchy process (AHP), fuzzy AHP, fuzzy logic, and rule-based expert systems. In addition, knowledge and experience must be captured from knowledge library experts. One of the most popular methods used to access and investigate knowledge is simulated by analysis with engineering software, for example, 
DEFORM, finite element, and other computer-aided engineering (CAE) software. However, such tools have rarely been used to predict the quality of cold forging. In addition, DEFORM ${ }^{\circledR}$ system is a time-consuming and knowledge base is limited as well as it does not have a mechanism to learn from experience. The relevant previous works are delineated in the next section.

The fastener starts from the preform forging and heading process, which consists of upsetting and/or extruding. The heading process can be accomplished via a high-speed operation. Moreover, it can comprise single or multiple processes. Raw material is normally in a round shape, whereas the material types can be mild steel, tool steel, stainless steel, and other modern materials. The combination of material shape and types can make the process more complex; in this scenario, a suitable punch and die set is required that may contain a simple set of the two punches and two dies up to the more complex set using five punches and five dies. The raw material must be prepared to match the volume of diecontrolled conditions as well as the relationship between the diameters of the wire rod of the upsetting process is significant. Cold heading proceeds with forward or backward extrusion; forward extrusion is used when the raw material is smaller than the die, and the length and diameter must be related. Backward extrusion is used to form nuts, sleeves, and rivets. Bolts, screws, and stepped shafts which are major components affecting the automotive industry supply chain are produced by forward extrusion (Ashari et al., 2018). However, present industry requires very short time production with high quality products and more complex shapes. Therefore, this study aims to determine the effects of cold forging criteria, including shape complexity, degree of formability, upsetting ratio, and extrusion ratio, and to develop the quality prediction modeling of a preform fastener for cold forging with fuzzy logic compared and verified with DEFORM simulation.

\section{Previous Works}

Traditionally, the cold forging process has been based on both experience-related expert knowledge and computer-assisted engineering. Cold forging is applied to several making products. Cold heading is used for deformation of the head and body of the fastener (Petrescu et al., 2002). However, difficulty and complexity are associated with preform fastener processes. The concept is to deform and reduce cross-sectional material into the near net shape until the final finished dimensions are exact and there is a good quality of surface finishing, particularly on the edges and corners, as well as very small and undercut areas, using progressive steps (Butdee and Khanawapee, 2015). Mathematical modeling is applied to simulate a material flow and search for optimum processing parameters. Petrescu et al. (2002) proposed a simulation of the fastener manufacturing process using the finite element method (FEM) for the upsetting process. Stephen and Vollertsen (2010) investigated the upsetting ratio for laser-based free-form heading and compared it with the conventional method. Mukhopadhyay (2014) studied the upsetting ratio during free cold forging for commercially pure aluminum, whereas Chengliang.Hu et al. (2017) proposed a combination of forward and backward extrusion for determining friction in cold forging of complex parts. Tiernan et al. (2005) presented the influence of die angle, reduction ratio, and die land on the extrusion force during the extrusion process by an experimental and finite element analysis of the cold extrusion of high-grade (AA1100) aluminum. The number of forging steps that depends on forging process design and the shape for each step also depends on the shape complex, material property, extrusion ratio, and upsetting ratio of the material.

Several works have been done on cold forging using DEFORM to analyze material flow behavior. Suthep and Uten (2015) proposed cold heading of stainless steel, which is a 
complicated process based on DEFORM simulation, and they compared the parameters of production processes to factory processes. Cheng et al. (2015) proposed a cold semiprecision forging process for the 5052-aluminum alloy multi-sprocket. DEFORM-3D was used to simulate the flow velocity fields in cold semi-precision forging. Moreover, Ciancio et al. (2015) presented a comparative study of different artificial intelligence techniques to design a high-performance predictive tool for forging operation and test the result using DEFORM-2D. However, despite these studies, the previous works on the cold heading have rarely been concerned with making decisions, particularly on multi-criteria decision making, or knowledge capturing for use in predicting future uncertain events. Presently, there are several methods applied to analyze scenarios, make decisions with multiple criteria, and develop predictions, but they are used in other domains. Fuzzy sets and Fuzzy Analytical Analytic Hierarchy Process (FAHP) are widely used to make decisions in the face of imprecise problems. Zimmermann (1978) and Mamdani and Assilian (1975) explained fuzzy theory, which was originally proposed by Zadeh (1965), as describing a vague phenomenon of life in a mathematical way. The fuzzy logic system includes fuzzification, a knowledge base, a fuzzy inference system (FIS), and a defuzzification process. Fuzzification is the process where the crisp values of both input and output variables existing in the real world are transformed into fuzzy values using membership functions. There are multiple types of membership functions, such as triangular, trapezoidal, Gaussian, bell-shaped, sigmoidal, and s-curve waveforms, that can be applied in practice (Bai et al., 2006). The widely used method in decision making for problems with multiple criteria is the AHP, which was established found by Saaty (1980). Putra et al. (2018) proposed a method of determining the quality of gemstones using FAHP. Butdee and Phuangsalee (2019) used AHP and fuzzy AHP to create a modeling dealing with uncertain risk for bus bodymanufacturing supply chains. As mentioned above, many studies are concerned with cold forging using AHP, FAHP, fuzzy logic, and DEFORM simulation, but none of them have combined fuzzy logic and DEFORM, which has made an important contribution to the work of academics and practitioners. Some articles have presented prediction modeling using artificial neural networks (Sadighi and Mohaddecy, 2013). Moreover, fuzzy logic is investigated as a good tool for searching to achieve optimization and prediction (Berawi et al., 2019).

\section{Research Method}

Fuzzy logic is adopted to determine the important factors of a preform fastener production process in multi-criteria decision making compared with DEFORM simulation. The number of heading steps is carried out under the aspect of design cost optimization. Quality and defects are considered in the preform heading process because of the rapid production process.

The research framework is designed to consist of input, process, and output. The input variables consist of five factors that influence the cold heading process, which are as follows: shape complexity (SC), degree of forgeability (DoF), upsetting ratio, extrusion ration (ER), and design of heading step. Such variables mainly affect the quality of preform fasteners. The quality can be explained in terms of a lack of defects, where defects include indentation, fractures, and surface imperfection. The fuzzy rule-based system is created as input and output membership functions, where there are five inputs and two outputs. The fuzzy rules are developed together with the FIS to provide all alternative decision making. The output carries a quality expectation based on the fuzziness concept. 


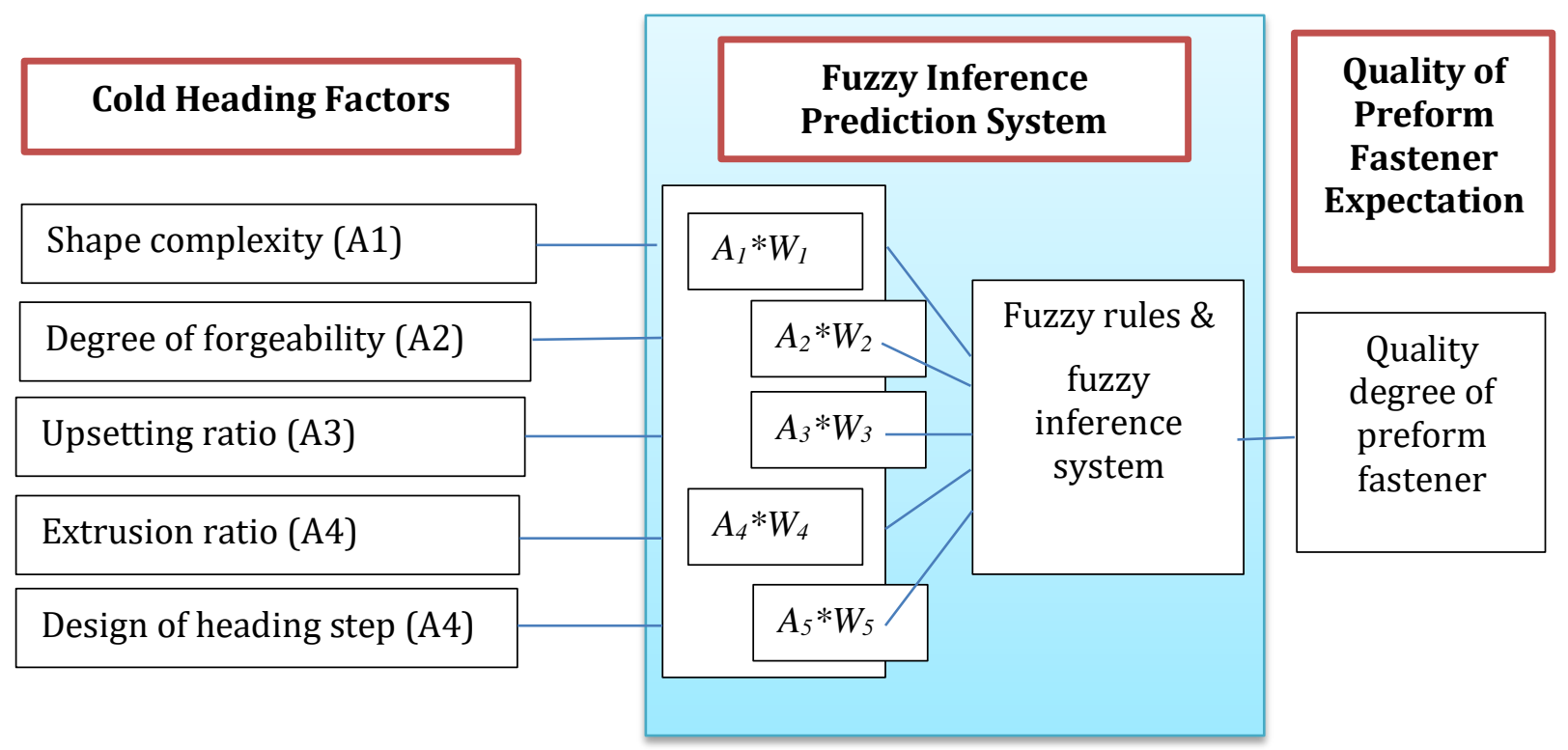

Figure 1 Framework of fuzzy logic for quality prediction of preform fastener deformation

\subsection{Shape Complexity}

Figure 2 shows the preform fastener classification, which includes the following categories: very simple, simple, medium, complex, and very complex. The very simple shape comprises a cylinder body and smooth head, whereas the simple shape shows a cylinder body with a slot head. Very simple fasteners are used for studs or rivets. The simple fastener shape is used for a simple screw. The medium shape represents a cylinder body with hexagonal head and slot or pocket. This type of fastener is used in the automotive industry. The complex shape represents a cylinder shape and hexagonal head with a flank and the presence or absence of a socket. This type of fastener is used for important parts fitting with heavy loads in the automotive industry, for example, for the engine, suspension, or body frame. The very complex shape comprises multi-step cylindrical body with hexagonal head and a flank with or without a socket.

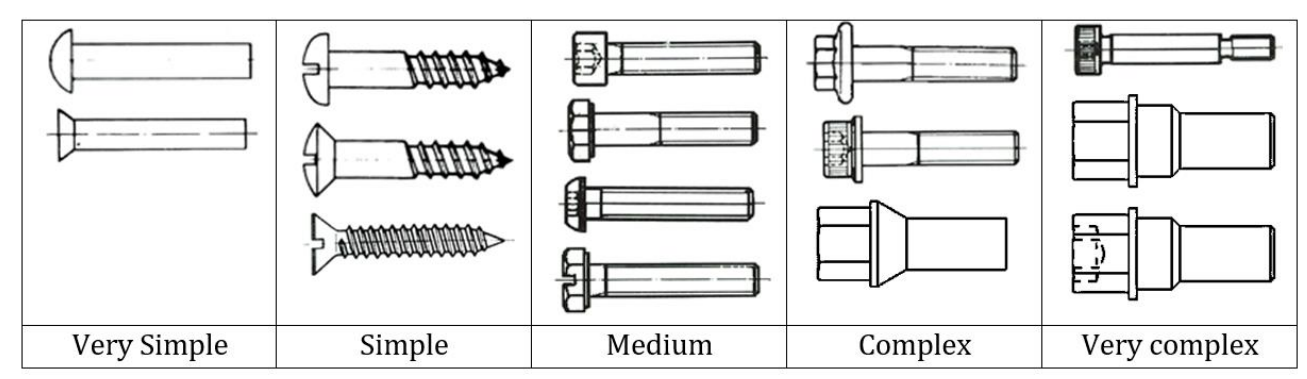

Figure 2 Fastener shape classification by complexity

\subsection{Degree of Forging}

The degree of forging (DoF), also called forgeability, represents the ability of material flow to be deformed in the die cavity. It is generally measured by the principle strain " $\varphi_{p}$." The cold forging process involves plastic permanent deformation, which retains its shape permanently. It is caused by sufficiently high shear stresses. Principle Strain is flow stress (Kstr), which means the strain hardening behavior of the material. The degree of forging involves direct deformation resistance (Kr), which is overcome during deformation. The DoF depends upon the chemical composition, crystalline structure, and heat treatment. 


\subsection{Upsetting Ratio}

The upsetting ability depends on the upsetting ratio (s), which shows the stock constraint deformation. The upsetting ratio is also known as upset forging, which is a bulkforming process where the effect of the pressure is on the longitudinal axis of the workpieces. It is commonly used in the application of mass production, such as for screws, rivets, and head bolts. It begins with the starting stock, which is a length of rod cut from round stock or wire coils. Permission deformations are used to consider the limitation of stock constraints in aspects of the extent of deformation and the upsetting ratio. If the stock constraint is over the limit constraint, buckling will occur (Tschaetsch, 2006). The upsetting ratio is the proportion between stock length and the initial diameter. The extrusion ratio is the reduction of the wire diameter and the workpiece. The decision can be divided into die types. If the reduction $\leq 35 \%$, one heading step operation is selected. In the case of trapped dies, one blow can be applied to reduction constraints $\leq 75 \%$.

\subsection{Extrusion Ratio}

The extrusion ratio (degree of extrusion) is the proportion of cross-section before and cross-section after deformation. Extrusion can cooperate with the cold heading process. It includes forward extrusion and backward extrusion. The extrusion ratio affects the quality of the cold forging process.

\subsection{Design of Heading Step}

The heading steps directly determine the forgeability and quality of the preform fastener. Practically, there are a maximum of five steps using five punches and five dies. The more heading steps are selected, the higher the quality that is achieved and the higher the cost that is incurred.

\section{Fuzzy Inference Prediction System}

This section presents the fuzzy logic prediction system applied to predict the quality of the preform fastener for enhancing bolt production in a variety of shapes and kinds of materials. The system consists of input, inference engine, and output. The input is concerned with influencing factors that affect the output. The cold heading is a process of preparing the preform fastener to make bolts, studs, and fasteners. The quality is generally considered by the precision; surface roughness (Ra); surface strength attributes, such as toughness, ductility, and crack resistance; and perfection of the preform which can be defined as bulging, metal not completely filling in the shank or head, or both. In this paper, two criteria are considered- $\mathrm{Ra}$ and perfection. The cold heading process capability is ranked as $0.8-6.3 \mu \mathrm{m}$ of $\mathrm{Ra}$. The input includes the four following variables: SC $\left(\mathrm{A}_{1}\right)$, forgeability or degree of forging $\left(A_{2}\right)$, upsetting ratio $\left(A_{3}\right)$, extrusion ratio $\left(A_{4}\right)$, and heading steps $\left(A_{5}\right)$ as shown in the Table 1.

Table 1 Variables and membership functions

\begin{tabular}{|c|c|c|c|c|c|}
\hline $\begin{array}{c}\text { Variable/ } \\
\text { Membership function }\end{array}$ & Maximum & 4 & & $\longrightarrow$ & Minimum \\
\hline Shape complexity $\left(A_{1}\right)$ & $\begin{array}{c}\text { Very } \\
\text { complex }\end{array}$ & Complex & Medium & Simple & Very simple \\
\hline Degree of forging $\left(\mathrm{A}_{2}\right)$ & 1 & 4 & & $\rightarrow$ & 0 \\
\hline Upsetting ratio $\left(\mathrm{A}_{3}\right)$ & 2.5 & & & $\longrightarrow$ & 0.7 \\
\hline Extrusion ratio $\left(\mathrm{A}_{4}\right)$ & 3.9 & 4 & & $\longrightarrow$ & 0.7 \\
\hline Heading step ( $\left.\mathrm{A}_{5}\right)$ & 5 & 4 & 3 & 2 & 1 \\
\hline
\end{tabular}


The membership function can be expressed as follows:

$$
A=\left(A_{1}, A_{2}, A_{3}, A_{4}, A_{5}\right) \Rightarrow Q \& C
$$

$\mathrm{Q}$ is quality, whereas $\mathrm{C}$ refers to cost.

Figure 3 shows the input variables, FIS, and output variables. There are five inputs and two outputs. The inputs are SC, upsetting ratio, extrusion ratio, degree of forgeability, and heading step. The outputs are quality and cost.

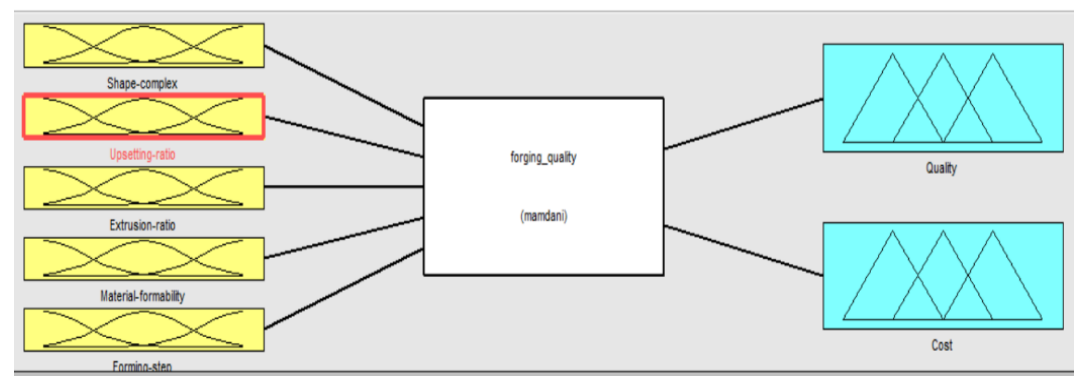

Figure 3 Input variables, FIS, and outputs

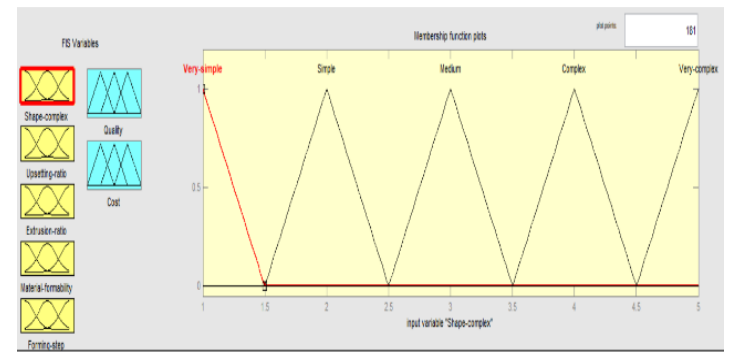

(a)

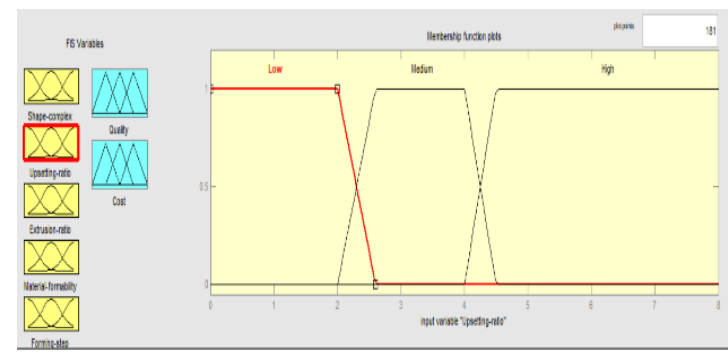

(b)

Figure 4 Input membership function: (a) shape complexity; (b) degree of forgeability

Figure 4 shows the input membership function. The SC is classified into the five following groups: very simple, simple, medium, complex, and very complex. This is shown in Figure 4a. Figure $4 \mathrm{~b}$ illustrates the membership function of the degree of forgeability. It is divided into the three following levels: low, medium, and high. Triangular is applied to the SC membership function, whereas the forgeability membership function uses trapezoid. Figure 5a shows the input membership function of the upsetting ratio, which is classified into the three following categories: low, medium, and high. Figure $5 b$ shows the input membership function of ER. It can be divided into the three following levels: low, medium, and high.

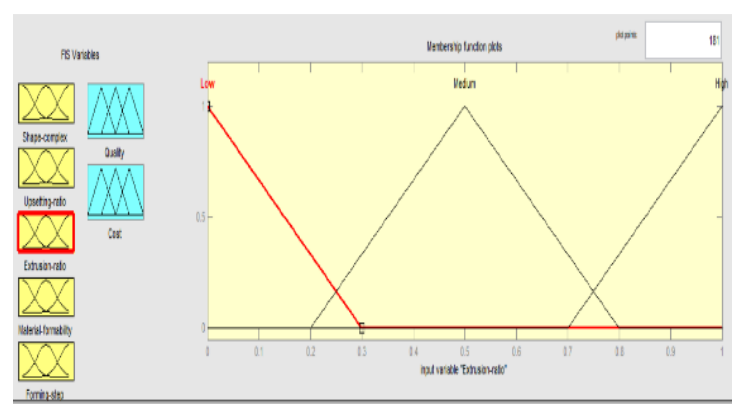

(a)

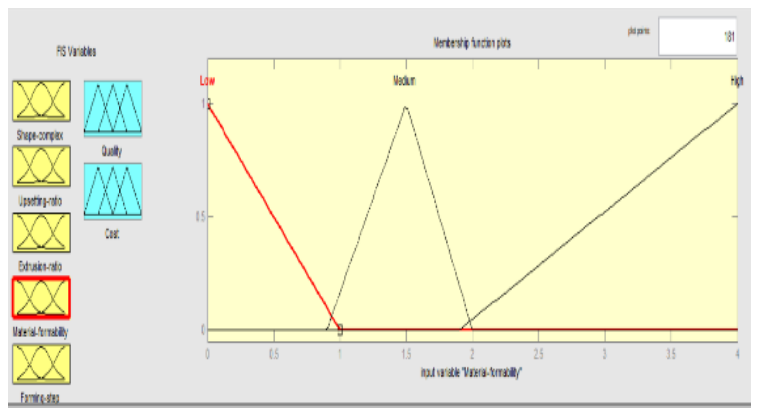

(b)

Figure 5 Input membership function: (a) upsetting ratio; (b) extrusion ratio 
Figure 6 shows the input membership function of the heading step. Practically, there are five steps. The number of heading steps affects both the quality and cost. The more heading steps that are chosen, the higher the quality received and the higher the cost will be. Therefore, the least number of heading steps should be selected as much as possible. Figure 7 shows the fuzzy rules for inference decision making of the quality and cost prediction. There are 675 rules in the fuzzy knowledge-based system.

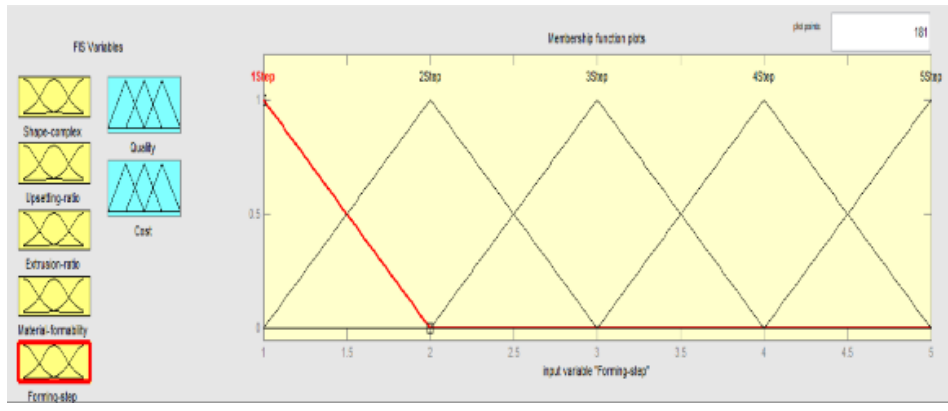

Figure 6 Input membership function of the heading step

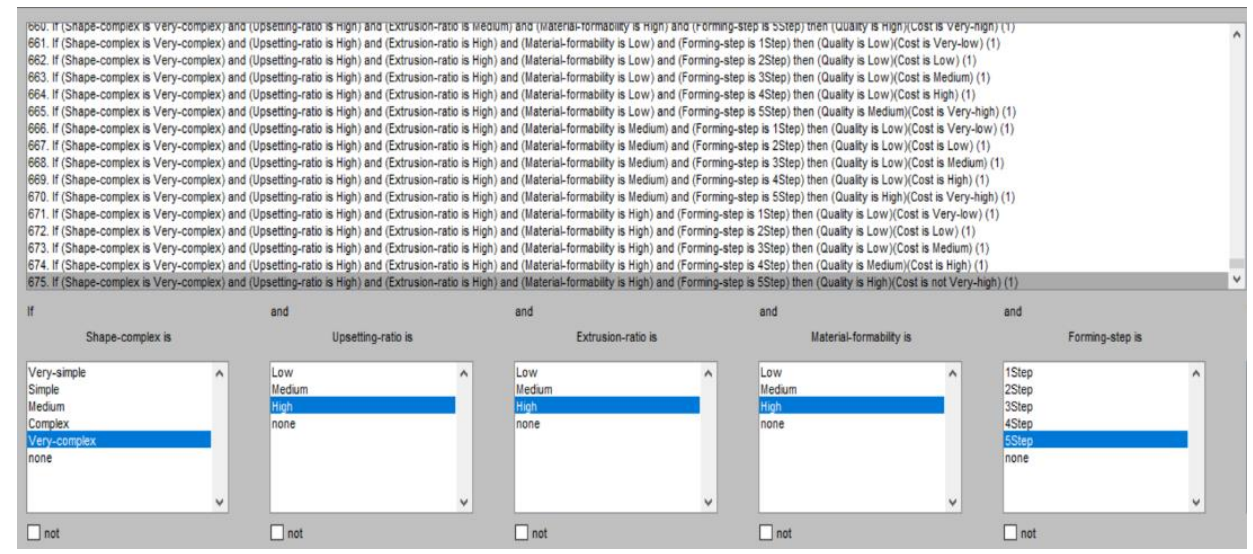

Figure 7 Fuzzy rules for inference decision making of the quality and cost prediction

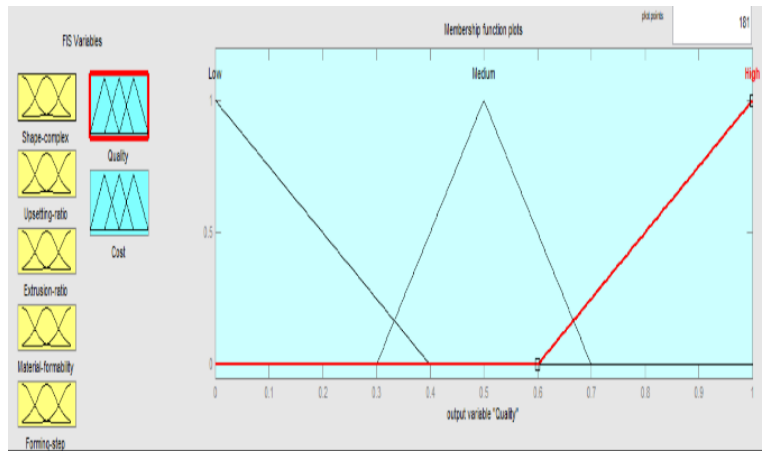

(a)

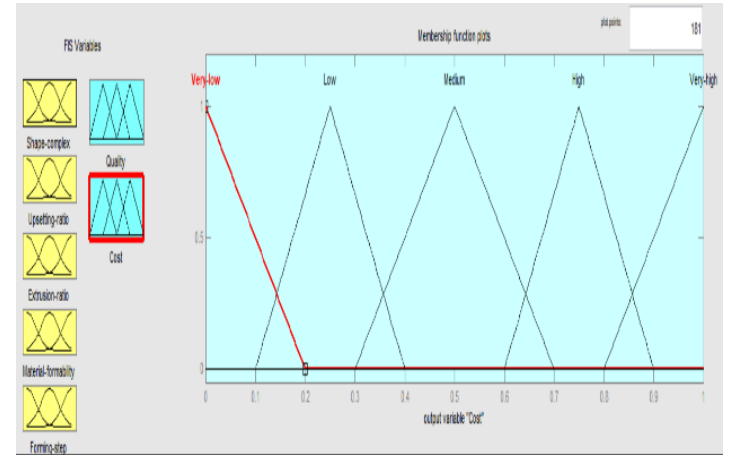

(b)

Figure 8 Output membership functions of the: (a) quality; and (b) cost

Figure 8 shows the output membership functions of the quality and cost. The quality membership function is divided into the three following levels: low, medium, and high. The rank is between 0 and 1, which means the quality level between $0 \%$ and $100 \%$. High quality is accepted over $70 \%$. The cost is ranked from 0 to 1 , using the same framework as for quality. 


\section{Results, Verification, and Discussion}

The result of the modeling is shown in Figure 9 as a rule viewer, which is simulated by MATLAB. This is a multi-criteria fuzzy set of five inputs and two outputs. An example of a case study showed that the cold forging complex with an upsetting ratio is 1.44 , and the extrusion ratio is 0.406 . Material formability or degree of forgeability is 1.59 . Four forging steps are selected. The result is expected to be 0.86 or $86 \%$, and the cost is quite expensive, at $75 \%$. The result is acceptable.

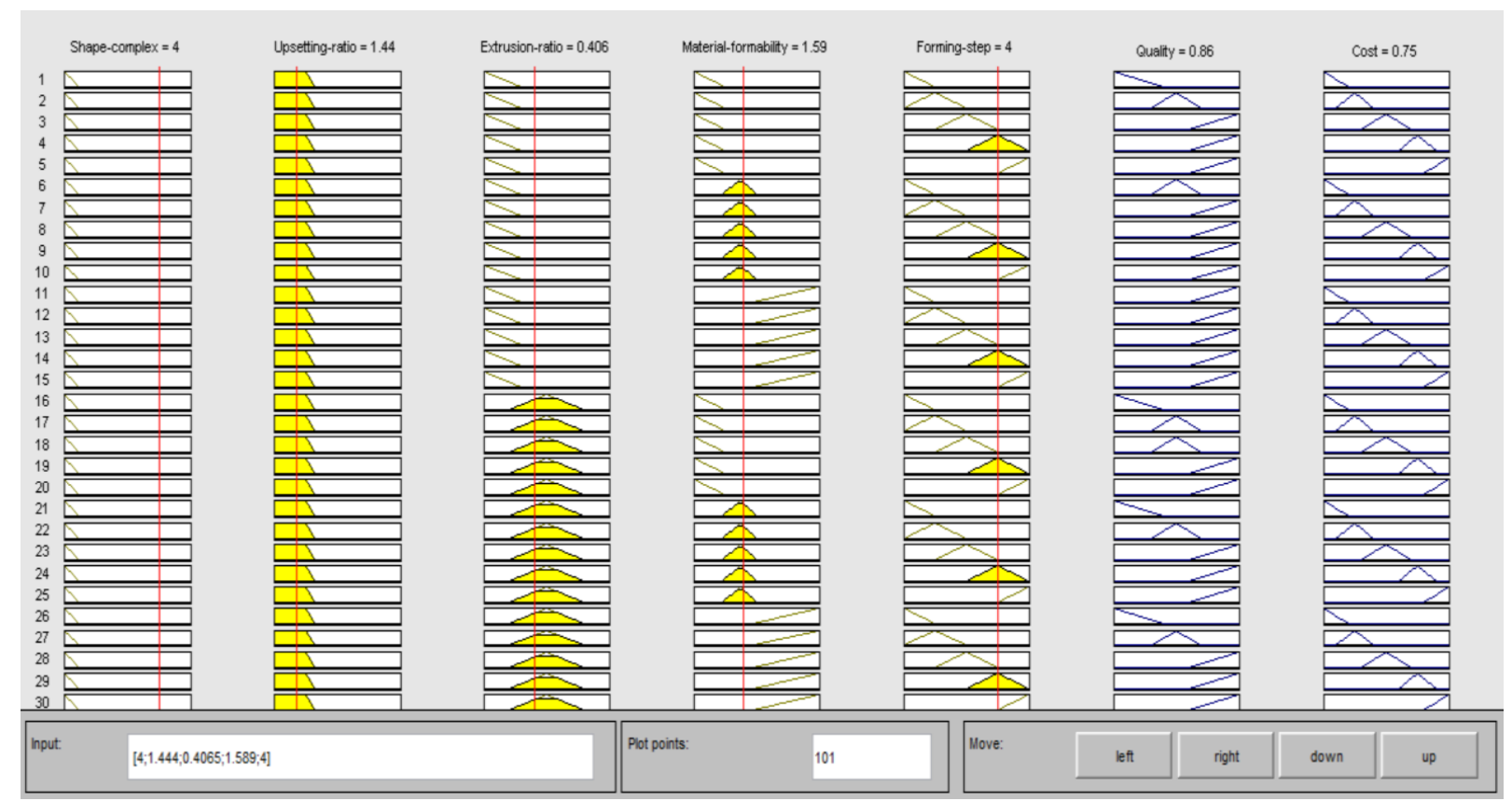

Figure 9 Rule viewer for sample FIS prediction

Figure 10 shows the result of the DEFORM simulation based on the condition of stainless graded 341 material. The first simulation is one-step forging. It shows that the completion is not finished. The forging force is linearly increased, and the load prediction is 54,000 Kilogram-force (kgf). The final result is bulging and failure. The problem has two aspects: the head is not finished, and the body is indented. The second simulation is twostep forging. It shows that the prediction load is linearly increased. The maximum load is $48,000 \mathrm{kgf}$ at one step. The force is increased sharply from the beginning and stays for a period of time. Then, the prediction load is dropped to zero between steps 1 and 2 . The prediction load is increased again at the beginning of the second step and finished at 70,000 kgf. The result is that the head and the body are not finished; the dies are still open. The third experiment involves three-step forging. The load is linearly increased at the beginning and dropped to zero and then increased again twice until the maximum load is attained at 56,000 kgf. There are 235 punching steps. The result is quite perfect and completely finished with a lower load applied. There is little defect occurring at the head of the fastener. The fourth experiment is four-step forging. The prediction load sharply increases at the beginning and varies four times. The maximum load is 53,000 kgf. The final result is quite perfect. The head and body are fulled with metal texture.

The result of modeling is simulated by MATLAB, whereas the material flow simulation is run on DEFORM. Both results head in the same direction. The fuzzy logic prediction system can be more understandable in scientific form and degree of quality and cost in percentages, which will assist in making decisions easily. In contrast, DEFORM is good at 
graphical representation and generating knowledge database on cold forging. The final formability is shown in different simulations. The final result shows that the complex shape should be performed by the five steps of the cold heading to achieve acceptable quality and cost.

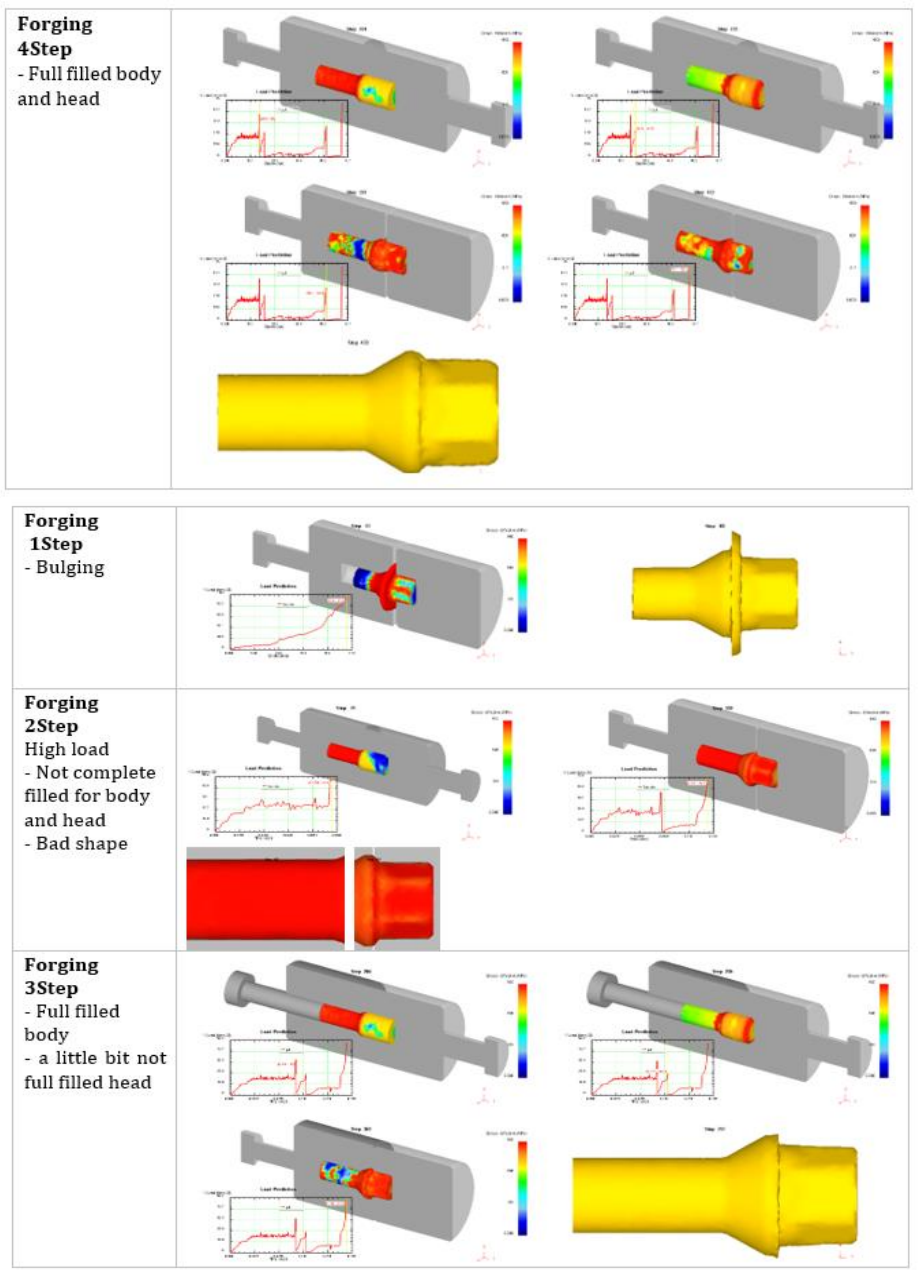

Figure 10 DEFORM simulation results

\section{Conclusions}

In this study, quality prediction of fastener processing using fuzzy logic was applied and presented with practical factor selection. The fuzzy approach can be used by the MATLAB toolbox. The prediction is shown as the percentage of successful forging. If the prediction quality is more than $70 \%$, it is acceptable. In the present results, the quality of completion and perfection are more than $90 \%$, which is satisfactory. The simulation experiment was verified using DEFORM. It was found that the two-step simulation generated the highest maximum load $(70,000 \mathrm{kgf})$, whereas the four-step simulation gave the lowest maximum load (53,000 kgf). The final result confirmed that four-step forging is workable, whereas five-step cold forging gives better quality.

\section{References}

Ashari, H., Yusoff, Y.M., Zamani, S.N.M., Talib, A.N.A., 2018. A Study of the Effect of Market Orientation on Malaysian Automotive Industry Supply Chain Performance. International Journal of Technology, Volume 9(8), pp. 1651-1657 
Bai, Y., Zhuang, H., Wang, D., 2006. Fundamentals of Fuzzy Logic Control-Fuzzy Sets, Fuzzy Rules and Defuzzifications. Advanced Fuzzy Logic Technologies in Industrial Applications, Volume 25, pp. 17-36

Berawi, M.A., Leviakangas, P., Muhammad, F., Sari, M., Gunawan., Yatmo, Y.A., Suryanegara, M., 2019. Optimizing Search and Rescue Personnel Allocation in Disaster Emergency Response using Fuzzy Logic. International Journal of Technology, Volume 10(7), pp. 1416-1426

Butdee, S., Khanawapee, U., 2015. Collaborative Knowledge for Analysis Material Flow of a Complex Long Stud using Multiple Stoke Cold Heading. IFIP Advances in Information and Communication Technology, Volume 459, pp. 102-109

Butdee, S., Phuangsalee, P., 2019. Uncertain Risk Assessment Modelling for Bus Body Manufacturing Supply Chain using AHP and Fuzzy AHP. Procedia Manufacturing, Volume 30, pp. 663-670

Cheng, W.J., Chi, C.Z., Wang, Y.Z., Peng, L.I.N., Zhao, R.H., Liang, W., 2015. 3D FEM Simulation of Flow Velocity Field for 5052 Aluminium Alloy Multi-Row Sprocket in Cold SemiPrecision Forging Process. Transactions of Nonferrous Metals Society of China, Volume 25(3), pp. 926-935

Ciancio, C., Citrea, T., Ambrogio, G., Filice, L., Musmanno, R., 2015. Design of a HighPerformance Predictive Tool for Forging Operation. Procedia CIRP, Volume 33, pp. 173178

Chengliang Hu, Qiang Yin, Zhen Zhao, 2017. A Novel Method for Determining Friction in Cold Forging of Complex Parts Using a Steady Combined Forward and Backward Extrusion Test. Journal of Materials Processing Technology, Volume 249, pp. 57-66

Mamdani, E.H., Assilian, S., 1975. An Experiment in Linguistic Synthesis with a Fuzzy Logic Controller. International Journal of Man-Machine Studies, Volume 7, pp. 1-13

Putra, D., Sobandi, M., Andryana, S., Gunaryati, A., 2018. Fuzzy Analytical Hierarchy Process Method to Determine the Quality of Gemstones. Advances in Fuzzy Systems, Volume 2018, pp. 1-6

Mukhopadhyay, A., 2014. A Systematic Approach to Determine the Impact of Upsetting Ratio During Free Cold Forging Operation of Commercially Pure Aluminium in Dry Condition. Procedia Materials Science, Volume 5, pp. 1281-1290

Petrescu, D., Savage, S.C., Hodgson, P.D., 2002. Simulation of the Fastener Manufacturing Process. Journal of Materials Processing Technology, Volume 125(126), pp. 361-368

Saaty, T.L.,1980. The Analytic Hierarchy Process. McGraw-Hill, New York, USA

Sadighi, S., Mohaddecy, R.S., 2013. Prediction Modelling for Industrial Naphtha Reforming Plant using Artificial Neural Network with Recurrent Layers. International Journal of Technology, Volume 4(2), pp. 102-111

Stephen, A., Vollertsen, F., 2010. Upset Ratios in Laser-Based Free form Heading. Physics Procedia, Volume 5(2), pp. 227-232

Suthep, B., Uten, K., 2015. Cold Forging Deformation Analysis for Stainless Material Using DEFORM. In: Proceedings of the 2015 IEEE IEEM, pp. 1277-1281

Tiernan, P, Hillery, M.T., Draganescu, B., Gheorghe, M., 2005. Modelling of Cold Extrusion with Experimental Verification. Journal Material Processing Technology, Volume 168(2), pp. 360-366

Tschaetsch, H., 2006. Metal Forming Practise, German: Springer Verlag Berlin Heidelberg Zadeh, L.A., 1965. Fuzzy Sets. Information and Control, Volume 8, pp. 338-352

Zimmermann, H.-J., 1978. Fuzzy Programming and Linear Programming with Several Objective Functions. Fuzzy Sets and Systems, Volume 1(1), pp. 45-55 